\title{
Exposição Solar Ocupacional e Câncer de Pele Não Melanoma: Estudo de Revisão Integrativa
}

Occupational Exposure to the Sun and Non-Melanoma Skin Cancer: Integrative Review

\author{
Exposición Solar Laboral y el Cáncer de Piel No Melanoma: Estudio de Revisión \\ Integradora
}

\author{
Albanita Gomes da Costa de Ceballos'; Solange Laurentino dos Santos ${ }^{2}$; Ana Catarina Alves e Silva ${ }^{3}$; Bruna Rafaele Vieira Pedrosa ${ }^{4}$; Mateus Morais \\ Aires Camara ${ }^{5}$; Sarah Luanne Silva ${ }^{6}$
}

\section{Resumo}

Introduçáo: $\mathrm{O}$ câncer de pele náo melanoma (CPNM) representa 25\% dos tumores registrados no Brasil, com baixa letalidade e elevada morbidade; acarreta despesas aos serviços de saúde; e afeta o bem-estar do indivíduo. Objetivo: Estudar a exposição solar ocupacional como fator predisponente para esse tipo de câncer. Método: Levantamento de artigos científicos completos nas bases de dados PubMed, LILACS e Science Direct. Os artigos selecionados foram catalogados e analisados de forma descritiva e crítica, de acordo com as particularidades de cada estudo. Resultados: Profissionais da jardinagem, da construção civil, trabalhadores agrícolas, da pecuária e pesca, devido à exposição diária e contínua à radiação ultravioleta (UV), têm maior chance de desenvolver CPNM. A exposição ocupacional em idade precoce, especialmente em indivíduos de pele clara e residentes de países próximos à linha equatorial, aumenta a chance de desenvolver câncer de pele. Conclusão: $\mathrm{O}$ principal fator de risco encontrado para CPNM foi a exposição à radiação UV e a exposição solar de caráter ocupacional. Os estudos pesquisados mostraram que há ocupaçóes mais propensas a desenvolver CPNM devido à exposição diária e contínua, como profissionais da construção civil e trabalhadores agrícolas, sendo a exposição ocupacional iniciada em idade mais precoce (inferior aos 30 anos) considerada de maior risco. Assim, reconhecendo a importância do conhecimento sobre os fatores de risco para a prevençáo do câncer e as dificuldades metodológicas encontradas nos artigos pesquisados, é importante a realização de mais estudos epidemiológicos que esclareçam a relação entre a exposição ocupacional e o CPNM.

Palavras-chave: Neoplasias Cutâneas/etiologia; Exposiçáo Ocupacional; Raios Ultravioleta; Revisão

\footnotetext{
Estudo vinculado a atividades do projeto Vigilância do câncer na Cidade do Recife: visibilizando os riscos e os fatores associados. O projeto foi aprovado no edital SGETS/MS No 28, de 22 de novembro de 2012, para o biênio 2013-2014, no Programa de Educaçáo para o Trabalho em Saúde/Vigilância em Saúde (PET/VS). Pedrosa, Camara, Silva, Santos e Ceballos recebem bolsa do Ministério da Saúde (MS) pelo PET/VS. Alves e Silva recebe bolsa de mestrado da Coordenaçáo de Aperfeiçoamento de Pessoal de Nível Superior (CAPES).

${ }^{1}$ Fonoaudióloga. Doutora em Saúde Pública. Professora-Adjunta do Departamento de Medicina Social e do Programa de Pós-Graduação Integrado em Saúde Coletiva da Universidade Federal de Pernambuco (UFP). Recife (PE), Brasil. E-mail: albanita.costa@ufpe.br.

${ }^{2}$ Cirurgiá-Dentista. Doutora em Saúde Pública. Professora-Adjunta do Departamento de Medicina Social e do Programa de Pós-Graduação Integrado em Saúde Coletiva da UFP. Recife (PE).E-mail: solange.lsantos@ufpe.br.

${ }^{3}$ Cirurgiä-Dentista. Mestranda do Programa de Pós-Graduaçáo Integrado em Saúde Coletiva da UFP. Recife (PE), Brasil. E-mail: anacatarina_alvesilva@yahoo.com.br.

${ }^{4}$ Graduanda em Odontologia na UFP. Recife (PE), Brasil. E-mail: brunarvpedrosa@gmail.com.

${ }^{5}$ Graduando em Medicina na UFP. Recife (PE), Brasil. E-mail: mairesufpe@gmail.com.

${ }^{6}$ Graduanda em Farmácia na UFP. Recife (PE), Brasil. E-mail: sarah.luannee@gmail.com.

Endereço para correspondência: Albanita Gomes da Costa de Ceballos. Departamento de Medicina Social da Universidade Federal de Pernambuco. Avenida da Engenharia, s/n, Bloco D, $1^{\circ}$ andar - Cidade Universitária. Recife (PE), Brasil. CEP: 50.740-600.E-mails: albanitagcceballos@gmail.com; albanita.costa@ufpe.br.
} 


\section{INTRODUÇÃO}

Câncer de pele é a neoplasia maligna mais comum em todo o mundo e sua incidência tem atingido caráter epidêmico ${ }^{1}$. Pode ser classificado em câncer de pele melanoma (CPM) e em câncer de pele não melanoma (CPNM). O CPM, apesar da elevada mortalidade, representa apenas 4\% dos cânceres da pele; e o CPNM, de baixa letalidade, corresponde a 90\% dos cânceres de pele e $25 \%$ de todos os tumores malignos registrados no Brasil, com estimativa para 2014 de 182 mil novos casos ${ }^{2,3}$. Entre os CPNM, estão o carcinoma basocelular (CBC) e o carcinoma espinocelular (CEC) ${ }^{4}$. Ambos têm origem de tecidos da epiderme; contudo o $\mathrm{CBC}$ é quatro vezes mais frequente que o $\mathrm{CEC}^{1,5}$.

A exposição a raios ultravioleta (UV) A e B é o principal fator de risco para gênese dos $\mathrm{CPNM}^{1,6}$. A exposição ocupacional de forma intermitente é um fator importante para o CBC, diferentemente do CEC, cuja exposição continuada é mais relevante. Considera-se que a exposiçáa cumulativa e excessiva durante os primeiros 10-20 anos de vida aumenta o risco de câncer de pele, o que é importante em regiôes com grande quantidade de crianças trabalhadoras ${ }^{7}$.

Considerando que a carga horária média de trabalho no Brasil é de 39,4 horas semanais ${ }^{8}$, pessoas que trabalham ao ar livre, como: agricultores, salva-vidas, trabalhadores da construção civil e professores de Educação Física, podem receber uma dose de radiação UV seis a oito vezes maior que trabalhadores de locais fechados?

Diante da alta incidência de CPNM e de seu grande impacto financeiro no sistema de saúde, associados à alta prevalência de trabalhadores ao ar livre no Brasil, este estudo tem como objetivo estudar a exposição solar ocupacional como fator predisponente para esse tipo de câncer.

\section{MÉTODO}

A construção desta revisão integrativa foi baseada na proposta de Ganong ${ }^{10}$. Na primeira etapa, foram feitas a identificação da questáo do estudo ou problematizaçáo e as buscas pelos descritores ou palavras-chave nas bases de dados selecionadas. Na segunda etapa, foram realizadas a seleção da amostra e a determinaçáo dos critérios de inclusão e exclusão, estabelecendo melhor qualidade e confiabilidade na seleção. $\mathrm{Na}$ terceira etapa, fizeram-se a categorização dos estudos, organização e sumarização das informaçôes dos artigos revisados. Na quarta etapa, foi feita a avaliação dos estudos. Na quinta etapa, foram conduzidas a discussão e a interpretação dos resultados. $\mathrm{Na}$ sexta e última etapa, apresentação da revisão integrativa e síntese do conhecimento.

A relação entre exposição solar ocupacional e CPNM foi a questão norteadora do estudo. A busca dos artigos foi feita nas bases de dados PubMed, LILACS (Literatura Latino-Americana e do Caribe em Ciências da Saúde) e Science Direct com resumos disponíveis e acessados na íntegra pelo meio on-line. Foram utilizados os descritores em inglês occupational cancer, carcinoma, basal cell, occupational health com base no DeCS (Descritores em Ciências da Saúde). Foram incluídos artigos completos publicados em português, inglês ou espanhol, que retratassem a temática do CPNM de origem ocupacional, devido à exposição solar, publicados nos últimos dez anos (2004 a 2013). A busca foi realizada no período de outubro a dezembro de 2013. Foram excluídos os artigos publicados antes do período delimitado, em outros idiomas, que retratassem outros tipos de câncer que não o CPNM, e cujo texto completo não estava disponível.

Os artigos foram fichados e catalogados de acordo com as seguintes categorias: título do artigo, base indexada, autores, Qualis da revista, ano de publicação, país, idioma, tipo de estudo, método, amostra, tratamento de dados e resultados. A análise dos artigos selecionados foi realizada de forma descritiva e crítica, de acordo com as particularidades de cada estudo.

\section{RESULTADOS}

No PubMed, foram encontrados 65 artigos; na LILACS, 97 artigos; e no Science Direct 68 artigos. Destes, de acordo com os critérios de inclusão, foram selecionados 8,5 e 1 trabalho, respectivamente. Os artigos que se repetiam foram considerados em apenas uma das bases de dados.

Quanto ao periódico de publicação, cada estudo foi publicado em uma revista diferente, com exceção de dois ${ }^{1,12}$. Conforme o Quadro 1, dez artigos já referem a temática ocupacional no próprio título. Apenas um tem sua publicação em uma revista de saúde pública, sugerindo uma maior frequência de publicaçóes sobre o tema em revistas específicas da área médica, como dermatologia e oncologia. Quanto ao ano de publicação, verificou-se que haviam sido publicados dois estudos no ano de 2007, um no ano de 2009, dois estudos no ano de 2010 e três estudos em cada um dos anos subsequentes.

Seis entre os 14 artigos se configuram como estudos de revisão (Quadro 2). Entre estes, três eram revisôes sistemáticas e um era uma revisão integrativa. O segundo tipo de estudo mais frequente foi o caso-controle, com quatro artigos; entre os quais, dois apresentavam descrição detalhada sobre a seleção dos casos e controles, na qual consideravam os casos indivíduos entre 20 e 70 anos de idade com diagnóstico confirmado de CBC e CEC, enquanto os controles foram obtidos por amostragem aleatória, realizada nas mesmas regiōes em que foram recrutados os casos, e estratificados por idade e sexo ${ }^{16,17}$. 
Quadro 1. Distribuição das referências incluídas no estudo segundo título, base de dados, periódico, ano de publicação, fator de impacto e país de origem

\begin{tabular}{|c|c|c|c|c|c|c|}
\hline $\mathbf{N}^{\circ}$ & Título & $\begin{array}{c}\text { Bases de } \\
\text { dados }\end{array}$ & Periódicos & Ano & $\begin{array}{l}\text { Fator de } \\
\text { Impacto }\end{array}$ & $\begin{array}{l}\text { País de } \\
\text { origem }\end{array}$ \\
\hline 1 & $\begin{array}{l}\text { Occupational skin cancer may } \\
\text { be underreported }{ }^{14}\end{array}$ & PUBMED & $\begin{array}{l}\text { Danish Med. } \\
\text { journal }\end{array}$ & 2013 & 0,92 & Dinamarca \\
\hline 2 & $\begin{array}{l}\text { Occupational Exposure to } \\
\text { Ultraviolet Radiation and Risk of } \\
\text { Non-Melanoma Skin Cancer in } \\
\text { a Multinational European Study'15 }\end{array}$ & PUBMED & Plos One & 2013 & 3,73 & $\begin{array}{l}\text { Hungria, } \\
\text { Romênia e } \\
\text { Eslováquia }\end{array}$ \\
\hline 3 & $\begin{array}{l}\text { Recognized Occupational Skin } \\
\text { Cancer in Denmark - Data From } \\
\text { the Last Ten Years"1 }\end{array}$ & PUBMED & $\begin{array}{l}\text { Acta dermato- } \\
\text { venereologica }\end{array}$ & 2013 & 3.487 & Suécia \\
\hline 4 & $\begin{array}{l}\text { The Relationship Between } \\
\text { Occupational Sun Exposure and } \\
\text { Non-Melanoma Skin Cancer }{ }^{4}\end{array}$ & PUBMED & $\begin{array}{l}\text { Deutsches } \\
\text { Ärzteblatt Int. }\end{array}$ & 2012 & 3.542 & Alemanha \\
\hline 5 & $\begin{array}{l}\text { Occupational skin cancer induced } \\
\text { by ultraviolet radiationand its } \\
\text { prevention' }\end{array}$ & LILACS & $\begin{array}{l}\text { British Journal of } \\
\text { Dermatology }\end{array}$ & 2012 & 3.759 & Inglaterra \\
\hline 6 & $\begin{array}{l}\text { Predictors of Basal Cell } \\
\text { Carcinoma in High-Risk Patients } \\
\text { in the VATC (VA Topical Tretinoin } \\
\text { Chemoprevention) Trial }{ }^{19}\end{array}$ & PUBMED & $\begin{array}{l}\text { Journal Investig. } \\
\text { Dermatology }\end{array}$ & 2012 & 6.193 & $\begin{array}{l}\text { Estados } \\
\text { Unidos }\end{array}$ \\
\hline 7 & $\begin{array}{l}\text { Fatores de risco para câncer da } \\
\text { pele não melanoma em Taubaté, } \\
\text { SP: um estudo caso-controle }{ }^{2}\end{array}$ & $\begin{array}{l}\text { SCIENCE } \\
\text { DIRECT }\end{array}$ & $\begin{array}{l}\text { Rev. Assoc Médica } \\
\text { Brasileira }\end{array}$ & 2011 & 0,77 & Brasil \\
\hline 8 & $\begin{array}{l}\text { Is occupational solar ultraviolet } \\
\text { irradiation a relevant risk factor } \\
\text { for basal cell carcinoma? A } \\
\text { systematic review and meta- } \\
\text { analysis of the epidemiological } \\
\text { literature }^{12}\end{array}$ & PUBMED & $\begin{array}{l}\text { British Journal of } \\
\text { Dermatology }\end{array}$ & 2011 & 3.759 & Inglaterra \\
\hline 9 & $\begin{array}{l}\text { Epidemiologia do carcinoma } \\
\text { basocelular }{ }^{20}\end{array}$ & LILACS & $\begin{array}{l}\text { Anais Brasileiros } \\
\text { de Dermatologia }\end{array}$ & 2011 & 0,62 & Brasil \\
\hline 10 & $\begin{array}{l}\text { UV-induced skin cancer at } \\
\text { workplace and evidence-based } \\
\text { prevention }{ }^{13}\end{array}$ & LILACS & $\begin{array}{l}\text { International } \\
\text { Archives of } \\
\text { Occupational and } \\
\text { Environmental } \\
\text { Health }\end{array}$ & 2010 & 1.539 & Alemanha \\
\hline 11 & $\begin{array}{l}\text { Occupational exposure to non- } \\
\text { artificial UV-light and non- } \\
\text { melanocytic skin cancer - a } \\
\text { systematic review concerning a } \\
\text { new occupational disease }{ }^{21}\end{array}$ & PUBMED & $\begin{array}{l}\text { Journal } \\
\text { Deutschen } \\
\text { Dermatologischen } \\
\text { Gesellschaft }\end{array}$ & 2010 & 1.403 & Alemanha \\
\hline 12 & $\begin{array}{l}\text { Occupation and skin cancer: } \\
\text { the results of the HELIOS-I } \\
\text { multicenter case-control study } 16\end{array}$ & PUBMED & BMC Public Health & 2007 & 2,08 & $\begin{array}{l}\text { Espanha, } \\
\text { Itália e } \\
\text { França }\end{array}$ \\
\hline 13 & $\begin{array}{l}\text { Occupation and keratinocyte } \\
\text { cancer risk: a population-based } \\
\text { case-control study }{ }^{17}\end{array}$ & PUBMED & $\begin{array}{l}\text { Cancer Causes } \\
\text { Control } \\
\text { Clinical Oncology }\end{array}$ & 2007 & 3.200 & $\begin{array}{l}\text { Estados } \\
\text { Unidos }\end{array}$ \\
\hline 14 & $\begin{array}{l}\text { Prácticas frente a la radiación UV } \\
\text { y características epidemiológicas } \\
\text { de un grupo de pacientes con } \\
\text { CBC en um centro de referencia } \\
\text { nacional en Colombia }{ }^{18}\end{array}$ & LILACS & $\begin{array}{l}\text { Revista } \\
\text { Colombiana de } \\
\text { Cancerología }\end{array}$ & 2009 & $\begin{array}{c}\text { Não } \\
\text { encontrado }\end{array}$ & Colômbia \\
\hline
\end{tabular}


Quadro 2. Descrição dos estudos incluídos no estudo segundo o tipo de estudo, população estudada e principais resultados

\begin{tabular}{|c|c|c|c|}
\hline $\mathbf{N}^{\circ}$ & $\begin{array}{l}\text { Tipo de } \\
\text { estudo }\end{array}$ & População estudada & Principais resultados \\
\hline 1 & Descritivo & $\begin{array}{l}\text { Registros do Conselho Nacional de } \\
\text { Acidentes de Trabalho (2000-2009) }\end{array}$ & $\begin{array}{l}\text { Localização dos tumores na face/couro } \\
\text { cabeludo. Jardinagem e construção civil foram } \\
\text { as ocupações com mais casos de CPNM, sendo } \\
\text { o CBC o mais frequente }\end{array}$ \\
\hline 2 & Caso-Controle & $\begin{array}{l}\text { Estudo de base hospitalar. } 618 \text { casos } \\
\text { e } 527 \text { controles (oriundos de cirurgia } \\
\text { geral, ortopedia e trauma) }\end{array}$ & $\begin{array}{l}\text { Os resultados do estudo não mostram } \\
\text { associação entre um risco aumentado de CPNM } \\
\text { e a exposição à radiação UV natural ou artificial } \\
\text { no local de trabalho }\end{array}$ \\
\hline 3 & $\begin{array}{l}\text { Descritivo } \\
\text { (Editorial) }\end{array}$ & $\begin{array}{l}\text { Análise de dados do Conselho } \\
\text { Nacional de Lesões Industriais (NBII) } \\
\text { da Dinamarca }\end{array}$ & $\begin{array}{l}\text { 66\% dos casos de CPNM foram associados à } \\
\text { exposição ocupacional a raios UV. Os demais } \\
\text { estavam associados ao trabalho com asfalto, } \\
\text { soldagem, alcatrão e óleo mineral, como } \\
\text { jardinagem e na construção }\end{array}$ \\
\hline 4 & $\begin{array}{l}\text { Revisão } \\
\text { Sistemática }\end{array}$ & $\begin{array}{l}\text { Revisão de artigos para a relação entre } \\
\text { exposição ocupacional a UV e risco } \\
\text { para CCE }\end{array}$ & $\begin{array}{l}\text { Há maior risco de } C E C \text { da pele }(O R=1,77) \\
\text { e de } C B C(O R=1,43) \text { entre as pessoas que } \\
\text { trabalham ao ar livre }\end{array}$ \\
\hline 5 & $\begin{array}{l}\text { Revisão de } \\
\text { Literatura }\end{array}$ & Não descreve metodologia & Não descreve resultados \\
\hline 6 & $\begin{array}{l}\text { Estudo de } \\
\text { coorte }\end{array}$ & $\begin{array}{l}\text { Acompanhou } 1.131 \text { pacientes que já } \\
\text { tiveram ao menos duas lesões de CBC } \\
\text { ou CEC em período de } 4 \text { anos }\end{array}$ & $\begin{array}{l}\text { Os fatores mais importantes para novo CBC } \\
\text { foram: o CBC prévio, idade, escolaridade, } \\
\text { sensibilidade solar, exposição ocupacional } \\
\text { ao sol. Sugere que o CBC está relacionado à } \\
\text { exposição ocupacional precoce ao sol antes dos } \\
30 \text { anos }\end{array}$ \\
\hline 7 & Caso-controle & $\begin{array}{l}\text { Estudo de base hospitalar com } 264 \\
\text { residentes em Taubaté, sendo } 132 \\
\text { casos e } 132 \text { controles. A seleção dos } \\
\text { controles foi por amostragem de } \\
\text { conveniência }\end{array}$ & $\begin{array}{l}\text { A exposição solar ocupacional foi referida por } \\
70,1 \% \text { e o tempo de trabalho ao sol variou de } \\
0 \text { a } 73 \text { anos. Este estudo associou os fatores de } \\
\text { risco ao CPNM e identificou o número de horas } \\
\text { de exposição solar não ocupacional }\end{array}$ \\
\hline 8 & $\begin{array}{l}\text { Revisão } \\
\text { sistemática e } \\
\text { metanálise }\end{array}$ & $\begin{array}{l}\text { Amostra composta por } 24 \text { artigos } \\
\text { (coorte e caso-controle) sobre } \\
\text { exposição ocupacional a raios UV e } \\
\text { CBC }\end{array}$ & $\begin{array}{l}\text { Trabalhadores ao ar livre têm } 40 \% \text { mais risco } \\
\text { de desenvolver } \mathrm{CBC} \text {. Foi apontada relação } \\
\text { inversa entre latitude e risco de CBC relatado } \\
\text { na literatura }\end{array}$ \\
\hline 9 & Revisão & Não descreve metodologia & Não descreve resultados \\
\hline 10 & $\begin{array}{l}\text { Revisão } \\
\text { integrativa }\end{array}$ & $\begin{array}{l}\text { Avaliou a associação de exposição } \\
\text { solar UV relacionado com o trabalho } \\
\text { para o desenvolvimento de câncer de } \\
\text { pele }\end{array}$ & $\begin{array}{l}\text { Aumento anual de aproximadamente } 10 \% \text { na } \\
\text { incidência da doença. Evidências de que o uso } \\
\text { em longo prazo de proteção solar impede o } \\
\text { aparecimento de câncer }\end{array}$ \\
\hline 11 & $\begin{array}{l}\text { Revisão } \\
\text { sistemática }\end{array}$ & $\begin{array}{l}\text { Estudos sobre a exposição ocupacional } \\
\text { à luz UV em trabalhadores ao ar livre } \\
\text { ou com tarefas internas/externas }\end{array}$ & $\begin{array}{l}\text { Relação entre a exposição aos raios UV } \\
\text { ocupacional e SCC foi estatisticamente } \\
\text { significante (OR entre } 1.5 \text { e } 4.3 \text { ) }\end{array}$ \\
\hline 12 & Caso-controle & $\begin{array}{l}\text { Foram estudados todos os casos de } \\
\text { CPNM registrados entre } 11 / 1989 \text { a } \\
\text { 06/1993, de seis regiões europeias, } \\
\text { com idades entre } 20 \text { e } 70 \text { anos. } \\
\text { Os controles foram obtidos por } \\
\text { amostragem aleatória, nas mesmas } \\
\text { regiões em que foram recrutados os } \\
\text { casos }\end{array}$ & $\begin{array}{l}\text { Considerou a história ocupacional. Maior } \\
\text { chance de } C B C \text { entre os maquinistas e } \\
\text { ferroviários bombeiros }(O R=4,55) \text {, agricultores } \\
\text { especializados }(O R=1,65) \text { e vendedores } \\
(O R=3,02) \text {, mineiros e pedreiros }(O R=7,96) \text {. As } \\
\text { ocupações de maior chance de } C E C \text { foram os } \\
\text { trabalhadores da construção }(O R=2,95) \text {, motor } \\
\text { estacionário e operadores de equipamentos } \\
(O R=5,31) \text { e pedreiros }(O R=2,55)\end{array}$ \\
\hline 13 & Caso-controle & $\begin{array}{l}\text { Foi realizado um estudo caso-controle } \\
\text { de base populacional de CBC e CEC } \\
\text { em New Hampshire }\end{array}$ & $\begin{array}{l}\text { Os homens foram associados a um maior risco } \\
\text { para o } C B C \text { entre trabalhadores de jardinagem }\end{array}$ \\
\hline 14 & Descritivo & $\begin{array}{l}\text { O estudo incluiu } 203 \text { pacientes com } \\
\text { diagnóstico de CBC confirmado por } \\
\text { exame histopatológico em um centro } \\
\text { de referência na Colômbia, no ano de } \\
2008\end{array}$ & $\begin{array}{l}\text { Sobre a exposição ocupacional a raios UV, } 50 \% \\
\text { declarou ter se exposto antes dos } 15 \text { anos, } 45 \% \\
\text { relataram exposição também entre } 15 \text { e } 30 \\
\text { anos e } 41 \% \text { após os } 30 \text { anos. Essas atividades } \\
\text { ocupacionais são principalmente relacionadas } \\
\text { ao campo: agricultura, pecuária e pesca e } \\
\text { comércio ao ar livre }\end{array}$ \\
\hline
\end{tabular}


A maioria dos artigos analisados foi publicada em Inglês e pelo menos metade das revistas apresentava alto fator de impacto $(50 \%$ publicados em periódicos com fator de impacto superior a 3,00).

Dos 14 artigos, nove foram realizados na Europa, dois no Brasil, dois nos Estados Unidos e um na Colômbia. Apenas três artigos fazem referência sobre a influência da cor da pele como fator de risco para o desenvolvimento do câncer de pele. Em um dos artigos realizados no Brasil, $39 \%$ da amostra possuía ascendência europeia e foi possível estimar um risco três vezes maior para indivíduos de pele mais clara ${ }^{2}$. Os estudos encontraram uma maior frequência da doença em pessoas com olhos e pele mais claros ${ }^{2,18}$.

Quanto à ocupação, sete artigos referem que trabalhadores ao ar livre são mais suscetíveis a desenvolver $\mathrm{CPNM}^{4,11,12,14,17,18,21}$. Segundo quatro artigos, os trabalhos com jardinagem e na construção civil são as ocupaçóes com maior frequência de indivíduos acometidos ${ }^{1,4,11,17}$. Além dessas ocupaçóes, são citados os trabalhados agrícolas, da pecuária, pesca, e mineradores, por apresentarem, além da exposição solar, outras exposições relacionadas à função exercida $^{17,18}$.

Dois artigos relatam que a exposição à radiação UV durante as atividades laborais é um fator de risco significativo para o desenvolvimento do $\mathrm{CEC}^{1,19}$. É razoável supor que os trabalhadores ao ar livre com uma longa história de exposição ocupacional a raios UV têm um risco aumentado de desenvolver CPNM.

\section{DISCUSSÃO}

Os estudos são convergentes ao afirmar que exposição ocupacional a raios UV é um fator de risco bem estabelecido para CEC. Em relação ao $\mathrm{CBC}$, as referências estudadas também assumem que há aumento de risco com exposição solar ocupacional, no entanto citam que esse risco é presumível, pois faltam pesquisas para se quantificar o risco ${ }^{1,4,11,13}$.

Devido à exposição diária e contínua, algumas ocupaçôes são mais propensas a desenvolver CPNM, como referidos em vários estudos, com destaque para os profissionais da jardinagem e da construção civil ${ }^{4,11,14,17}$. Em menor proporção, porém ainda relevante, estão os trabalhadores agrícolas, da pecuária e pesca e mineiros, por apresentarem, além da exposição solar, outras exposições relacionadas à função ${ }^{16,18}$.

Em relação aos fatores de risco, o CPNM é mais frequente em homens, provavelmente devido ao não uso de protetor solar e por serem maioria entre os que trabalham ao ar livre. No entanto, um estudo transversal observou maior prevalência em mulheres (65\%), talvez por esse grupo procurar mais as equipes de saúde. Já em outro estudo, uma coorte com seguimento de 1.131 pacientes, conclui-se que a exposição solar ocupacional precoce (antes dos 30 anos) é mais importante que a $\operatorname{tardia}^{19}$. Dados de um estudo de prevalência ratificam essa constatação, na medida em que $50 \%$ dos pacientes com CPNM declararam ter tido exposição solar ocupacional antes dos 15 anos $^{18}$.

A localização geográfica tem notável importância quanto à exposiçáo solar, podendo ser considerada como um fator de risco para o desenvolvimento desse tipo de câncer. Estudos demonstram que a proximidade com a linha do equador é um fator de risco para o CPNM. Mesmo em áreas não cortadas pelo equador, como em países como Austrália e Estados Unidos, observa-se que a incidência do CEC aumenta quanto menor for a latitude ${ }^{1}$.

Quanto à localização primária do tumor, a face mostra-se como a principal área acometida, já que o CPNM aparece predominantemente em regióes do corpo que sofrem exposiçấo frequente ao sol e normalmente não recebem nenhum tipo de proteção. Outros estudos ainda referem escápula, couro cabeludo e dorso das mãos como locais muito acometidos ${ }^{1,11,14,18,19}$.

A diminuição da exposição ocupacional ao sol do meio-dia, a utilização de toldos nos horários de trabalho ao ar livre, o uso adequado de proteção individual, como vestuário, chapéus e protetores solares, são propostos pelos estudos como formas eficientes de prevenção. Contudo a falta de proteção apropriada, seja por indisponibilidade do empregador, seja por não uso das medidas de proteção, acarreta aumento de exposição do trabalhador à radiação UV.

Embora haja consenso de que exposição ocupacional a raios UV aumente o risco de CPNM, alguns problemas metodológicos são apresentados nos estudos pesquisados. A ausência de dados objetivos quanto à quantidade da exposição a raios UV, viés de memória do pesquisado, aplicação de protocolos náo validados ou reconhecidos pela comunidade científica, dificuldade em discernir a exposição ocupacional da exposição recreativa e ainda erros de classificação quanto à exposiçáo ocupacional, considerando a ocupação no momento do diagnóstico do câncer e não levando em conta a história ocupacional, podem levar a medidas equivocadas da relação entre exposição ocupacional e a doença. Dessa forma, é importante considerar a necessidade de estudos epidemiológicos metodologicamente mais rigorosos para maior esclarecimento dessa relação.

\section{CONCLUSÃO}

Os CPNM têm grande importância no cenário da saúde pública, uma vez que causam elevada morbidade e são um dos cinco tipos de câncer que mais oneram os sistemas de saúde. O principal fator de risco para CPNM é a exposição a raios UV, e a exposição solar de caráter ocupacional é também fator de risco independente, tanto para CEC como para CBC. 
Os estudos pesquisados mostraram que, para a exposição diária e contínua, há ocupaçóes mais propensas a desenvolver CPNM, como profissionais da jardinagem, da construção civil, trabalhadores agrícolas, da pecuária e pesca. O momento da exposição é um ponto importante, sendo a exposiçáo ocupacional iniciada em idade mais precoce (inferior aos 30 anos) considerada de maior risco que em idades mais avançadas. Indivíduos de pele clara e residentes de países próximos à linha equatorial também têm risco aumentado e a região da cabeça e pescoço, por ser mais exposta ao sol, é frequentemente a localização primária desse tipo de tumor, recomendando-se, também para CPNM de origem ocupacional, medidas preventivas como uso de filtro solar e proteçáo física contra o sol.

Considerando a importância do conhecimento sobre os fatores de risco para a prevenção do câncer e as dificuldades metodológicas encontradas nos artigos pesquisados, é importante a realização de mais estudos epidemiológicos que esclareçam a relação entre a exposição ocupacional e o CPNM.

\section{CONTRIBUIÇÕES}

Albanita Gomes da Costa de Ceballos e Ana Catarina Alves e Silva trabalharam na concepção, elaboração e revisão do artigo; Solange Laurentino dos Santos trabalhou na concepção e revisão do artigo; Bruna Rafaele Vieira Pedrosa, Mateus Morais Aires Camara e Sarah Luanne Silva trabalharam na concepção e elaboração do artigo.

\section{Declaraçáo de Conflito de Interesses: Nada a Declarar.}

\section{REFERÊNCIAS}

1. Diepgen TL, Fartasch M, Drexler H, Schimitt J. Occupational skin cancer induced by ultraviolet radiation and its prevention. Br J Dermatol. 2012 Aug; 167(Suppl 2):76-84.

2. Ferreira FR, Nascimento LFC, Rotta O. Fatores de risco para câncer da pele não melanoma em Taubaté, SP: um estudo caso-controle. Rev Assoc Med Bras. 2011;57(4):431-7.

3. Instituto Nacional do Câncer José Alencar Gomes da Silva. Estimativa 2014: incidência de câncer no Brasil. Rio de Janeiro: Inca; 2014.

4. Fartasch M, Diepgen LT, Schmitt J, Drexler H. The relationship between occupational sun exposure and non-melanoma skin cancer: clinical basics, epidemiology, occupational disease evaluation, and prevention. Dtsch Arztbl Int. 2012 Oct;109(42):715-20.

5. Azulay RD. Dermatologia. Rio de Janeiro: Guanabara Koogan; 2008.

6. Oliveira LM, Glauss N, Palma A. Habits related to sun exposure among physical education teachers working with water activities. An Bras Dermatol. 2011 Jun;86(3):445-50.

7. Instituto Brasileiro de Geografia e Estatística. Pesquisa nacional por amostra de domicílios: síntese de indicadores 2012: tabelas completas: rendimento [Internet]. [Rio de Janeiro]: IBGE. [acesso em 2013 dez 20]. Disponível em: http://www.ibge.gov.br/home/estatistica/populacao/ trabalhoerendimento/pnad2012/sintese_defaultpdf_ rendimentos.shtm.

8. Instituto de Pesquisa Econômica Aplicada. Carga horária de trabalho: evolução e principais mudanças no Brasil [Internet]. [Brasília, DF]: Ipea; 2009. [acesso em 2013 dez 20]. Disponível em: http://www.simpesc.org.br/ wp-content/uploads/2011/12/arq_24_red.pdf.

9. Holman CD, Gibson IM, Stephenson M, Armstrong BK. Ultraviolet irradiation of human body sites in relation to occupation and outdoor activity: field studies using personal UVR dosimeters. Clin Exp Dermatol. 1983 May;8(3):269-77.

10. Ganong LH. Integrative reviews of nursing research. Res Nurs Health, 1987; 10(1):1-11.

11. Carøe TK, Ebbehøj EN, Wulf CH, Agner T. Recognized occupational skin cancer in Denmark: data from the last ten years. Acta Derm Venereol. 2013 May;93(3):369-71.

12. Bauer A, Diepgen TL, Schmitt J. Is occupational solar ultraviolet irradiation a relevant risk factor for basal cell carcinoma? A systematic review and meta-analysis of the epidemiological literature. Br J Dermatol. 2011 Sep;165(3):612-25.

13. Kütting B, Drexler H. UV-induced skin cancer at workplace and evidence-based prevention. Int Arch Occup Environ Health. 2010 Dec;83(8):843-54.

14. Carøe TK, Ebbehøj EN, Wulf CH, Agner, T. Occupational skin cancer may be underreported. Dan Med J. 2013 May;60(5):A4624.

15. Surdu S, Fitzgerald EF, Bloom MS, Boscoe FP, Carpenter DO, Haases RF, et al. Occupational exposure to ultrviolet radiation and risk of non-melanoma skin cancer in a multinational european study. PloS One. 2013;8(5):1-9.

16. Suárez B, Abente-López G, Martínez C, Navarro C, Tormo JM, Rosso $S$, et al. Occupation and skin cancer: the results of the HELIOS-I multicenter case-control study. BMC Public Health, 2007;7: 1-13.

17. Marehbian J, Colt SJ, Baris D, Stewart P, Stukel AT, Spencer KS, et al. Occupation and keratinocyte cancer risk: a population-based case-control study. Cancer Causes Control. 2007 Oct;18(8):895-908.

18. Sánchez G, Nova J, Arias N. Prácticas frente a la radiación ultravioleta y características epidemiológicas de un grupo de pacientes con carcinoma basocelular en un centro de 
referencia nacional en Colombia. Rev Colomb Cancerol. 2010 Sept;14(3):144-51.

19. Dyer KR, Weinstock AM, Cohen TS, Rizzo AE, Bingham SF. predictors of basall cell carcimonia in high-risk patients in the VATTC (VA Topical Tretinoin Chemoprevention) trial. J Invest Dermatol. 2012 Nov;132(11):2544-51.
20. Chinem VP, Miot HA. Epidemiologia do carcinoma basocelular. An Bras Dermatolol. 2011 Apr;86(2):292-305.

21. Schmitt J, Diepsent T, Bauer A. Occupational exposure to non-artificial UV-light and non-melanocytic skin care: a systematic review concerning a new occupational disease. J Dtsch Dermatol. 2010 Apr;8(4):250-64. 


\begin{abstract}
Introduction: Non-melanoma skin cancer (NMSC) represents about 25\% of all tumors registered in Brazil. Although it presents low lethality, the high morbidity carries great costs to health services and affects the wellbeing of the individual. Objective: To study the occupational sun exposure as a predisposing factor for this type of cancer. Method: A search for completed scientific articles was done using PubMed, LILACS and Science Direct database. Selected articles were cataloged and analyzed descriptively and critically, according to the particularities of each study. Results: Professionals from gardening, construction, farm workers, livestock and fisheries, due to daily and continuous exposure to UV radiation, have a greater chance of developing cancer. Occupational exposure at an early age, especially in those with clear skin and residents of countries near the equator, increases the chance of developing skin cancer. Conclusion: The main risk factor found for NMSC was the exposure to UV radiation and occupational exposure to sun. The studies surveyed showed that it's more likely to develop occupational NMSC due to the continuous and daily exposure, as is the case for professionals in the construction and agricultural field, and the occupational exposure initiated at an earlier age (less than 30 years) is considered the greatest risk. Therefore, considering the importance of the knowledge about the risk factors, for cancer prevention and the methodological difficulties encountered in articles surveyed, it is important to conduct more epidemiological studies to clarify the relationship between occupational exposure and NMSC.
\end{abstract}

Key words: Skin Neoplasms/etiology; Occupational Exposure; Rayos Ultravioleta; Review

\title{
Resumen
}

Introducción: El cáncer de piel no melanoma (CPNM) representa el $25 \%$ de los tumores reportados en Brasil, de baja mortalidad y alta morbilidad; implica gastos a los servicios de salud y afecta el bienestar del paciente. Objetivo: Estudiar la exposición solar laboral como factor predisponente para este tipo de cáncer. Método: Determinar artículos científicos completos en las bases de datos PubMed, LILACS y Science Direct. Los artículos seleccionados fueron catalogados y analizados de forma descriptiva y crítica, de acuerdo a las particularidades de cada estudio. Resultados: Los profesionales de la jardinería, de la construcción civil, del área agrícola, de la ganadería y los de la pesca, debido a la exposición diaria y continuada a la radiación UV, tienen una mayor probabilidad de desarrollar cáncer de piel no melanoma (CPNM). La exposición laboral a una temprana edad, especialmente en las personas de piel clara y los residentes de países cerca de la línea ecuatorial, aumenta la probabilidad de tener cáncer de piel. Conclusión: El principal factor de riesgo encontrado para CPNM fue la exposición a la radiación UV y la exposición solar de carácter laboral. Los estudios mostraron que hay ocupaciones que tienen más probabilidades de desarrollar CPNM debido a una exposición diaria y continua, como por ejemplo, profesionales de construcción civil y trabajadores agrícolas, donde la exposición laboral iniciada a una temprana edad (menos de 30 años) fue considerada la de mayor riesgo. Entonces, teniendo en cuenta la importancia del conocimiento sobre los factores de riesgo para la prevención del cáncer y las dificultades metodológicas encontradas en artículos estudiados, es importante llevar a cabo más estudios epidemiológicos para aclarar la relación entre la exposición ocupacional y CPNM.

Palabras clave: Neoplasias Cutâneas/etiología; Exposición Profesional; Rayos Ultravioleta; Revisión 